\title{
Preventing Override Trip Algorithm based on Quantum Entanglement in Coal Mine High-Voltage Grid
}

\author{
Xinliang Wang ${ }^{\mathrm{a},}$, Zhigang Guo ${ }^{\mathrm{a}}$, Qianhui Yang ${ }^{\mathrm{b}}$, and Jianing Zou ${ }^{\mathrm{a}}$ \\ ${ }^{a}$ School of Physics and Electronic Information Engineering, Henan Polytechnic University, Jiaozuo, 454000, China \\ ${ }^{b}$ School of Electrical Engineering and Automation, Henan Polytechnic University, Jiaozuo, 454000, China
}

\begin{abstract}
In the traditional preventing override trip system, the preventing override trip issue is still not resolved very well because of the transmission delay problem. Based on the characteristic of quantum entanglement, a quantum preventing override trip algorithm is proposed, which can allow the power monitoring system to quickly obtain the switch's over current fault information and effectively prevent override trip of the coal mine high-voltage grid. The simulation results show that the quantum override trip algorithm uses less time to obtain fault information of all switches and saves more time to finish protection setting when compared with traditional algorithms. So, the quantum preventing override trip algorithm can better solve the problem of transmission delay and can effectively improve the reliability of preventing override trip system.
\end{abstract}

Keywords: coal mine high-voltage grid; quantum entanglement; over current faults; preventing override trip

(Submitted on January 29, 2018; Revised on March 3, 2018; Accepted on April 20, 2018)

(C) 2018 Totem Publisher, Inc. All rights reserved.

\section{Introduction}

There are many factors in coal mine underground trip, which mainly includes improper selection of the current transformer, un-matched short circuit current scale [7], improper voltage protection [6], improper protection setting value, which cannot correctly identify short circuits [4], and so on.

Many preventing override trip schemes have been proposed in China to target the problem of override trip, which usually appears in the comprehensive protection device of coal mines, but these schemes still have some problems. In order to ensure the safety of the underground power supply system, the literature [9] proposed the differential protection scheme based on fiber communication. It can better determine fault positions, however, it does not prevent override trip. The literature [5] proposed a new preventing override trip scheme. In this scheme, the high switches with cascade relationships are connected by a communication cable. When short-circuit fault occurs, all the switches on the short-circuit line will detect the short circuit current, and the switches that detected the short-circuit fault will send the locking signal to its superiors. This scheme is highly dependent on the cable. Because of the complex environment of the power supply system and the heavy electromagnetic interference, the delay time of the locking signal in long-distance transmission is longer, and the reliability of communication cannot be guaranteed. The literature [12] used the digital substation to prevent override trip. All the comprehensive protection devices are connected to the digital substation host. When the short circuit fault occurs in the line, the fault information in the GOOSE message will be transmitted to the substation host. Based on the cascade relationship of comprehensive protection devices, the host can distinguish between internal faults and external faults. When the internal fault is detected, the switch that is nearest to the fault will trip. The scheme has a high requirement for a communication system, and fault of the communication system network will result in expansion of the scope of the power failure or even lead to collapse of the entire system.

Aimed at the problems in the above schemes, quantum communication will be introduced in this paper to prevent override trip and solve the problem of large transmission delay by fault information. In 1993, Bennett proposed a scheme to

\footnotetext{
* Corresponding author.

E-mail address: junci158@163.com
} 
realize quantum teleportation based on the characteristic particles entanglement [1]. In 1997, Bouwmeester realized the first teleportation [2] that can construct a quantum channel by using an entangled photon. The literature $[3,8,10,11]$ respectively proposed multi-particle quantum teleportation, continuous variable of quantum teleportation and controlled quantum teleportation. In the literature [13,14], Zhou Nanrun constructed the communication protocol at link layer based on the characteristic of quantum entanglement, which could effectively improve the performance of the communication protocol.

Based on quantum entanglement, information can be transferred efficiently and speedily, however, studies on quantum preventing override trip scheme still has not been reported. In the quantum preventing override trip scheme, the quantum teleportation cannot be used to transmit fault information directly because the use of the classical information channel will cause large transmission delays and will affect the reliability of the quantum preventing override trip scheme.

This paper proposes a new preventing override trip algorithm based on quantum entanglement. It will establish the quantum channel between the power monitoring system and the comprehensive protection device, and the power monitoring system can distinguish whether there exists the short-circuit faults in the comprehensive protection devices. Then, the fault location can be judged according to the topological structure of the power supply system, and trip accidents can be avoided by setting different action delays to the corresponding devices. Furthermore, in this algorithm, even if there is a short jam in the communication link because of congestion, the transmission of the fault information will not be affected.

\section{Over-current fault detection algorithm based on quantum entanglement}

Quantum entanglement as three basic characteristics: (1) Quantum transfer is instantaneous; (2) Quantum transfers without knowing where the other side is in advance; (3) Transfer process will not be impeded by any obstacle. Overall, quantum does not need time in the process of information transmission except the time to establish quantum channels. By introducing the quantum entanglement in the underground preventing override trip system, the system reliability will be improved.

In coal mine high-voltage power grids, the optical fiber line can not only be used to set the switch state and the protector setting of the switch by the power monitoring system, but can also be used to get the state parameters of the switch protection device in a periodic polling manner. When the fiber is in the process of getting the state and set parameters, the fiber channel state is busy; when there is no data transfer in the fiber, the fiber channel state is free. In this paper, when the fiber channel state is free, it will be used to distribute EPR particles between the power monitoring system and protection device to achieve the short circuit fault location. The specific process is shown in Figure 1.

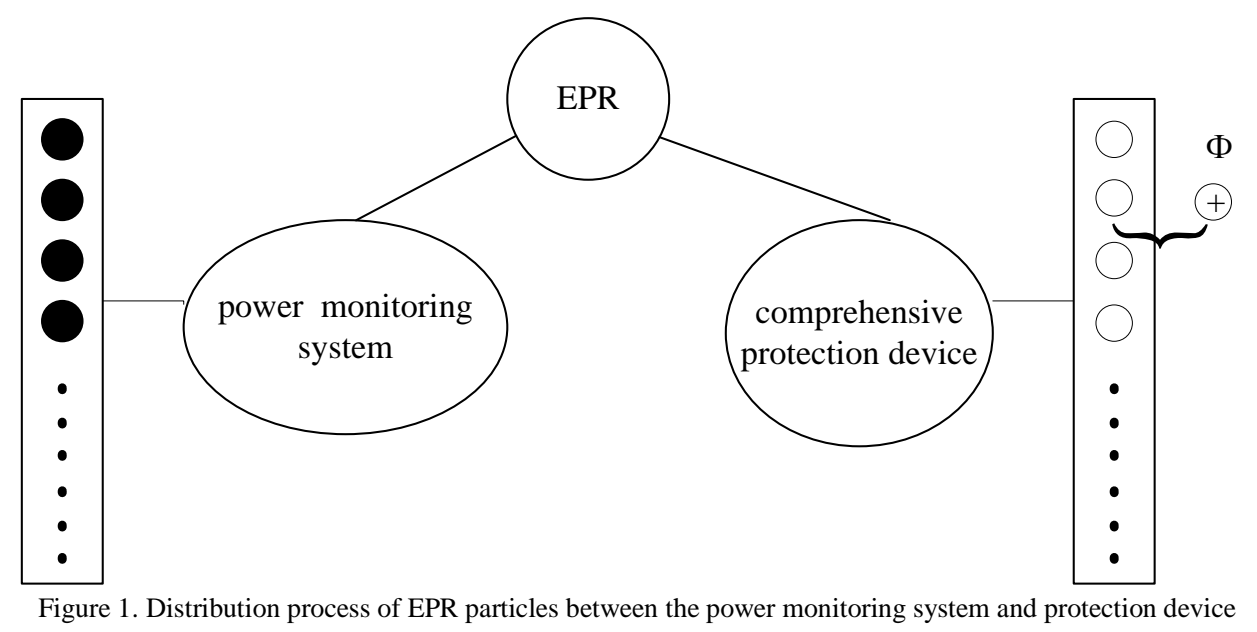

1) When the fiber channel state is free, EPR Center will prepare pair $M$ particles in the entangled state. Then, the entangled particles are respectively allocated to the power monitoring system and comprehensive protection device. Assuming the entangled state of the $i$ th particles pair between the power monitoring system and comprehensive protection device, it is described as $\left|\psi^{-}\right\rangle=\frac{1}{\sqrt{2}}(|0\rangle|1\rangle-|1\rangle|0\rangle)$.

2) At the initial moment, the comprehensive protection device determines whether the current detected is more than the action value by the periodic time interval $T$. If in the $i$ th measurement period, the current comprehensive protection device detects that the current is more than the action value and requires a trip, the comprehensive protection device will prepare a 
particle $|\varphi\rangle$, and $|\varphi\rangle=\frac{1}{\sqrt{5}}|0\rangle+\frac{2}{\sqrt{5}}|1\rangle$. Then, at the moment $t=i^{*} T$, use bell basis $\left|\psi^{ \pm}\right\rangle=\frac{1}{\sqrt{2}}(|0\rangle|1\rangle \pm|1\rangle|0\rangle)$ $\left|\varphi^{ \pm}\right\rangle=\frac{1}{\sqrt{2}}(|0\rangle|0\rangle \pm|1\rangle|1\rangle) \quad$ to achieve joint measurement of the particle $|\varphi\rangle$ and $\left|\psi^{-}\right\rangle$. The specific process is as shown in Equation 1 .

$$
\begin{aligned}
& |\varphi\rangle \otimes\left|\psi^{-}\right\rangle=\frac{1}{2}\left[\left|\psi^{-}\right\rangle\left(-\frac{1}{\sqrt{5}}|0\rangle-\frac{2}{\sqrt{5}}|1\rangle\right)+\left|\psi^{+}\right\rangle\left(-\frac{1}{\sqrt{5}}|0\rangle+\frac{2}{\sqrt{5}}|1\rangle\right)\right. \\
& \left.+\left|\varphi^{-}\right\rangle\left(\frac{2}{\sqrt{5}}|0\rangle+\frac{1}{\sqrt{5}}|1\rangle\right)+\left|\varphi^{+}\right\rangle\left(-\frac{2}{\sqrt{5}}|0\rangle+\frac{1}{\sqrt{5}}|1\rangle\right)\right]
\end{aligned}
$$

3) At the moment $t=i * T+\Delta t(\Delta \mathrm{t}<<\mathrm{T})$, the power monitoring system measures the particle $i$ that is ahead in the power monitoring system after ensuring the comprehensive protection device finished joint measurement. The measurement results of the power monitoring system are as follows: If the quantum state of the particle is one of $-\frac{1}{\sqrt{5}}|0\rangle-\frac{2}{\sqrt{5}}|1\rangle$, $-\frac{1}{\sqrt{5}}|0\rangle+\frac{2}{\sqrt{5}}|1\rangle, \quad \frac{2}{\sqrt{5}}|0\rangle+\frac{1}{\sqrt{5}}|1\rangle$ and $-\frac{2}{\sqrt{5}}|0\rangle+\frac{1}{\sqrt{5}}|1\rangle$, as shown in Table 1, then the power monitoring system can determine that the protector has an over-current fault; if the quantum state of the particle $i$ is $|0\rangle$ or $|1\rangle$, then it indicates that in this period of time interval $T$, the comprehensive protection device does not conduct a joint measurement. Thus, it can be determined that there is no over current fault in the interval $T$.

Table 1. The quantum measurement results of power monitoring system when there exists over current fault

\begin{tabular}{|c|c|}
\hline $\begin{array}{c}\text { Quantum state of comprehensive protection device when } \\
\text { appearing faults }\end{array}$ & Probability of quantum state When appearing fault \\
\hline$-\frac{1}{\sqrt{5}}|0\rangle-\frac{2}{\sqrt{5}}|1\rangle$ & $1 / 4$ \\
\hline$-\frac{1}{\sqrt{5}}|0\rangle+\frac{2}{\sqrt{5}}|1\rangle$ & $1 / 4$ \\
\hline$\frac{2}{\sqrt{5}}|0\rangle+\frac{1}{\sqrt{5}}|1\rangle$ & $1 / 4$ \\
\hline$-\frac{2}{\sqrt{5}}|0\rangle+\frac{1}{\sqrt{5}}|1\rangle$ & $1 / 4$ \\
\hline
\end{tabular}

\section{Preventing override trip algorithm based on the quantum entanglement}

In this paper, preventing override trip algorithm is proposed based on quantum entanglement characteristics. The algorithm can make the power monitoring system obtain the fault state information of each protector with less time, and it can provide a set of preventing override trip schemes based on the centralized controlling of the quantum power monitoring system. To prevent the override trip, the quantum module of the power monitoring system will finish the monitoring and measurement of every quantum corresponding to each protector in the periodic time interval $T$. If the quantum state is $|0\rangle$ or $|1\rangle$, it indicates that there does not exist an over-current fault in the protector; if the quantum state is one of $-\frac{1}{\sqrt{5}}|0\rangle-\frac{2}{\sqrt{5}}|1\rangle$, $-\frac{1}{\sqrt{5}}|0\rangle+\frac{2}{\sqrt{5}}|1\rangle, \quad \frac{2}{\sqrt{5}}|0\rangle+\frac{1}{\sqrt{5}}|1\rangle$ and $-\frac{2}{\sqrt{5}}|0\rangle+\frac{1}{\sqrt{5}}|1\rangle$, it indicates that there exists an over-current fault in the protector. According to the network topology structure and the fault state information of all protectors, the algorithm will quickly locate the over current protector, distribute the correct setting of protector, and improve the reliability of preventing override trip system.

\subsection{The principle of preventing override trip algorithm based on quantum entanglement}

The principle of preventing override trip algorithm based on quantum entanglement is shown in Figure 2. In Figure 2, when the short circuit fault occurs at the end of the line directly controlled by the $20 \#$ switch, we assume that the detected fault currents of the 20\#, 19\#, 16\#, 15\#, 9\#, 8\#, 2\#, 1\# switches are greater than the protection setting value. 
The power monitoring system can detect the switches with the over-current fault in the current period by the overcurrent fault detection algorithm based on quantum entanglement. According to the network topology of the power supply system and the fault state of the protector, the power monitoring system can specifically identify the switch needed to trip and quickly configure the appropriate setting values.

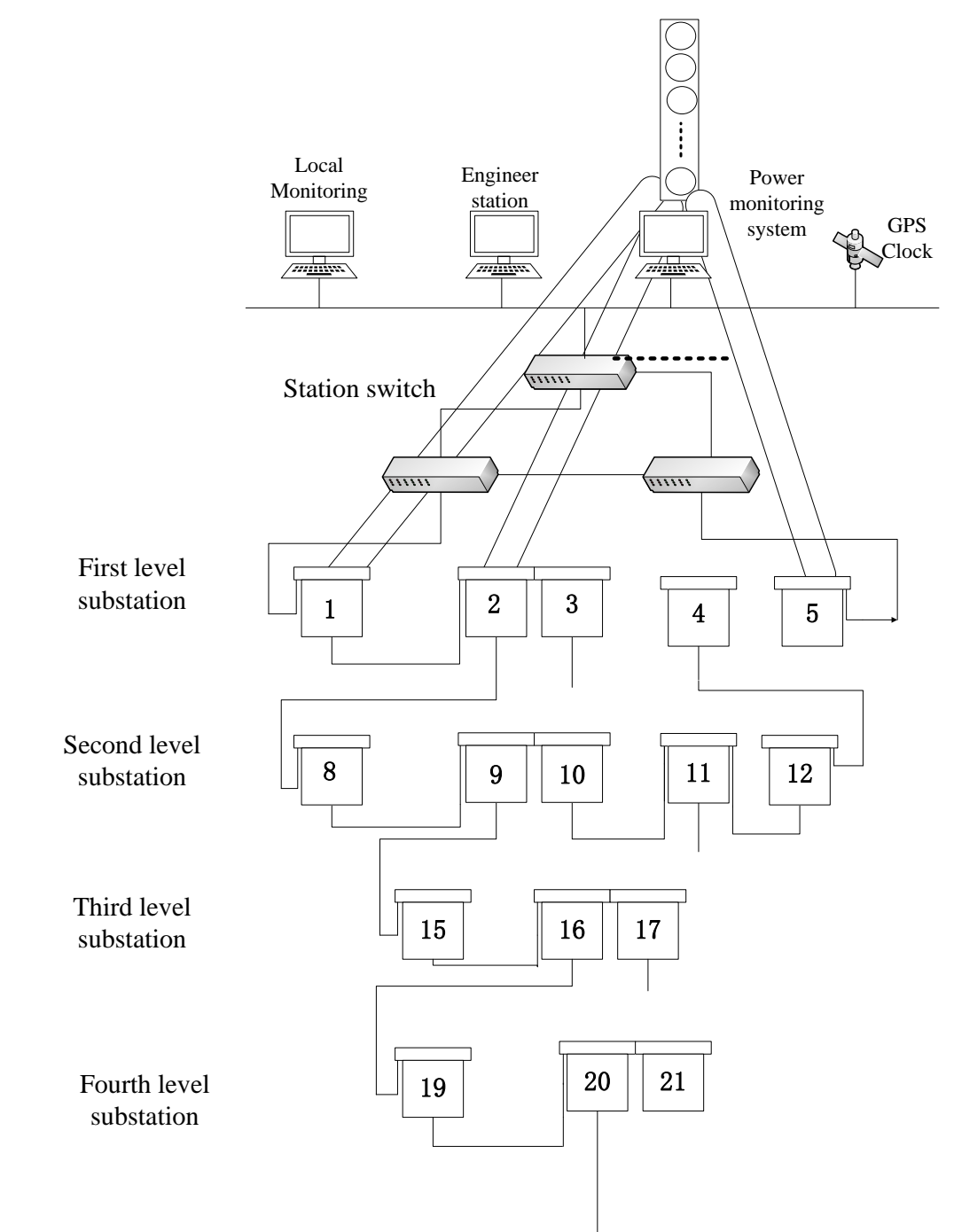

Figure 2. The principle of preventing override trip algorithm based on quantum entanglement

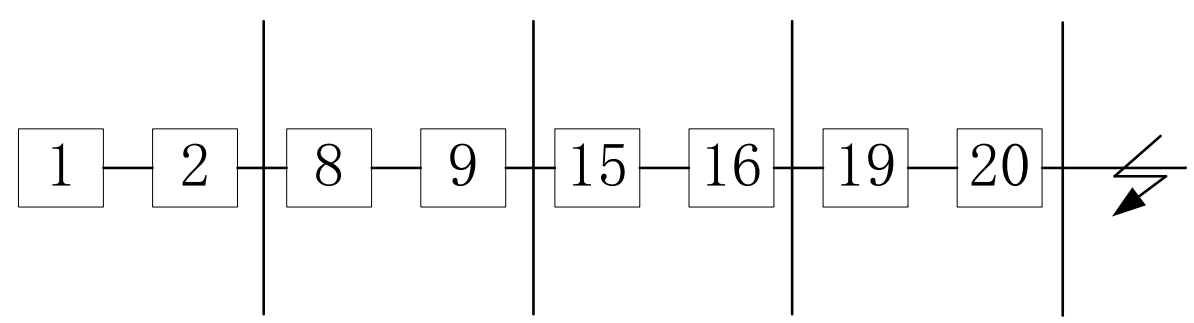

Figure 3. The short circuit fault information of protectors

As is shown in Figure 3, when there is a short circuit fault in the line directly controlled by the $20 \#$ switch, all the corresponding switches in Figure 3 will detect short circuit faults. If the fault occurs in the line directly controlled by the 16\# switch, there will be no current in the $20 \#$ switch. So, the power monitoring system is able to determine that the line directly controlled by the $20 \#$ switch has a fault. Therefore, according to the supply correlation of the switches, the short circuit fault line can correctly be located. 
When the short circuit fault occurs in the line directly controlled by the $20 \#$ switch, the power monitoring system will quickly modify the setting value of the corresponding switches. First of all, trip commands are sent out to the $20 \#$ switch, and the trip delay is set to 0. Assuming that the delay difference of each level is described as $r$, then the 16\#, 9\#, and 2\# switch trip delay is respectively set to $r, 2 r$ and $3 r$. In $r$ seconds, if the fault is not removed, the 16\# switch will trip immediately; in 2r seconds, if the fault is still not removed, the 9\# switch will trip immediately; in $3 \mathrm{r}$ seconds, if the fault is still not removed, the $2 \#$ switch will trip immediately. By quickly setting the different delay levels, the override trip problem can be improved to a greater extent. In a coal mine high-voltage grid, the tripping time allowed by the superior power supply department is limited. In the traditional preventing override trip scheme, it takes a long time for the power monitoring system to obtain the fault information of all high voltage switches. Thus, the delay time used to prevent override trip will be shortened, further limiting the coal mine high-voltage grid scale. While preventing override trip algorithm based on quantum entanglement is introduced, the time to get the fault information of all the high voltage switches will greatly be reduced, and more time can be reserved to set the switch delay at different levels. Even if the system scale becomes large, it can better solve the problems of the override tripping problem.

\subsection{Performance Analysis}

Assuming that the tripping time allowed by the superior power supply department is $T_{u}$, in the traditional preventing override trip scheme, the time to transfer fault information from the switch to the power monitoring system is described as $t_{1}$. Assuming there are $n$ switches in the coal mine high-voltage grid, the time to get the fault information of all the switch is $n * t_{1}$. In preventing override trip system, the time can be used to set the switch delay is described as $\tau$ and $\tau=T_{u}-n t_{1}$. To quantum prevent override trip system, the transmission time of over current fault information of the switches is instantaneous. In each period, the measurement of all the entangled particles relative to the protectors will be finished, and the fault information of every switch will be obtained by the power monitoring system. In quantum preventing override trip system, the measurement of entangled particles does not need network transmission, and the time to finish the measurement is mainly determined by the performance of the power monitoring system. By introducing parallel measurement mechanisms into the power monitoring system, measurement time $T$ of the fault information of obtaining $n$ switches can be greatly reduced, and it is $\tau=T_{u}-T$.

\section{Simulation}

Assuming $T_{u}=0.4 \mathrm{~s}, T=0.01 \mathrm{~s}$, and $t_{1}=0.004 \mathrm{~s}$, the delay time interval of each level is set to $r$. In traditional preventing override trip scheme, if $r=0.12 \mathrm{~s}$, the highest level of coalmine high-voltage grid that can be achieved is $N_{1}$. Assuming $x=\left\lfloor\frac{T_{u}-n t_{1}}{r}\right\rfloor=\left\lfloor\frac{0.4-0.004 * n}{0.12}\right\rfloor$, then $N_{1}=\left\{\begin{array}{l}x+1, x \geq 0 \\ 0, x<0\end{array} ;\right.$ In the quantum preventing override trip algorithm, the highest level of coalmine high-voltage grid that can be achieved is $N_{2}$ and $N_{2}=\left\lfloor\frac{T_{u}-T}{r}+1\right\rfloor=4$. The relationship of $n$ and the highest level of coalmine high-voltage grid is shown in Figure 4. The horizontal axis is the number of switches $n$, and the vertical axis is the highest level of coalmine high-voltage grid.

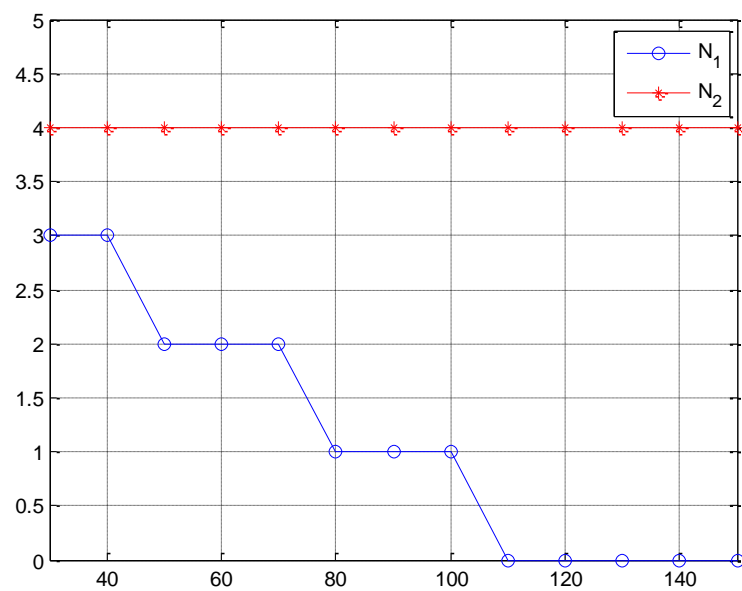

Figure 4. The relationship of $n$ and highest level of coalmine high-voltage grid. 
As is shown in Figure 4, in a traditional scheme, with an increase in the number of switches, it will take more time for the power monitoring system to obtain short-circuit fault information of all switches and the time to set the delay will be shorter. Eventually it will cause the highest level of coalmine high-voltage grid to reduce gradually along with the increasing number of switches. However, in the preventing override trip algorithm based on quantum entanglement, no matter how many switches there are, the power monitoring system can quickly obtain short circuit fault information of all switches by using the instantaneous transmission characteristics of quantum entanglement, and it can make the system reserve more time to finish the over-current setting. So, even if the number of switches increases, the highest level of coalmine high-voltage grid will remain unchanged.

Assuming $n=100, T_{u}=0.2 \sim 0.5$, in a traditional scheme, $N_{1}=\frac{T_{u}-n t_{1}}{r}+1=\frac{T_{u}-0.48}{0.12}$; in the preventing override trip algorithm based on the quantum entanglement, $N_{2}=\frac{T_{u}-T}{r}+1=\frac{T_{u}-0.01}{0.12}+1$. The details are shown in Figure 5. The horizontal axis is $T_{u}$, and the vertical axis is the highest level of the coalmine high-voltage grid.

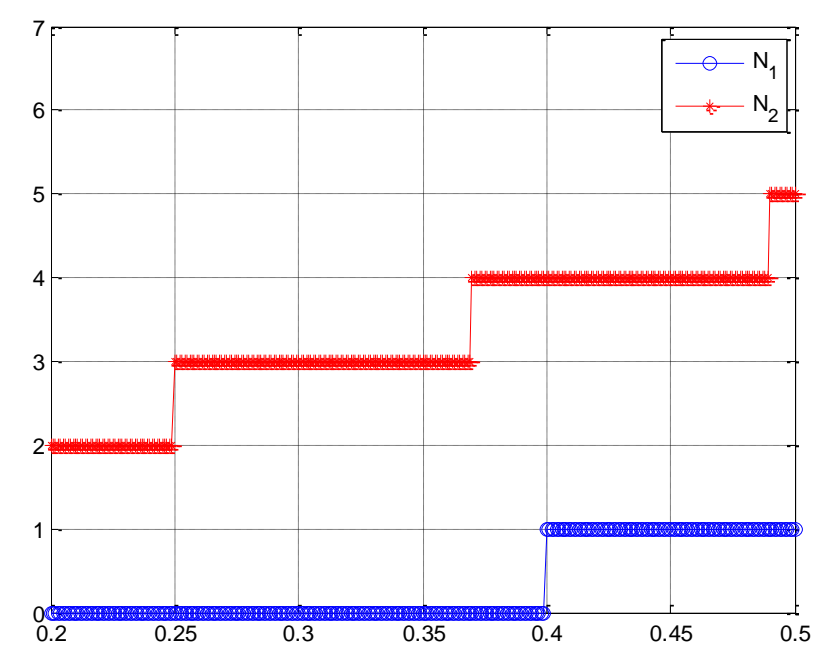

Figure 5. The relationship of the $T_{u}$ and highest level of coalmine high-voltage grid.

As shown in Figure 5, the trip time provided by the superior power supply department is shorter, and the levels that can realize the override protection is less. Obviously, the highest level of coalmine high-voltage grid in preventing override trip system based on the quantum entanglement is larger than the highest level in the traditional preventing override trip system. That is to say, even the tripping time provided by the superior power supply department is smaller, and the preventing override trip scheme based on quantum entanglement can meet the requirements of the switches level.

Compared with the traditional preventing override trip scheme, preventing override trip algorithm based on quantum entanglement is more suitable for large-scale coal mines. It can better meet the requirements of preventing override trip and can ensure the reliability of preventing override trip system. At the same time, the access to fault information of all switches is implemented by pre-assigned quantum particles. Even if there exists fault in the fiber communication network, it does not affect the power monitoring system obtainment of short circuit fault information because the entangled quantum particles have been pre-assigned in the power monitoring system and each comprehensive protection device in the free time of the fiber communication network. So, the preventing override trip system based on quantum entanglement owns a stronger fault tolerance and higher reliability for fiber communication fault.

\section{Conclusions}

In the preventing override trip algorithm based on the quantum entanglement, the time taken to obtain fault information of all switches by the power monitoring system has been greatly reduced. Accordingly, more time can be reserved to set different delay times for the switches at different levels, and it is more suitable for large-scale coal mines. Experimental results show that, compared with the traditional preventing override trip scheme, our method can better meet the requirements of preventing override trip and can ensure the reliability of preventing override trip system. 


\section{Acknowledgements}

This paper is supported by the Doctor Fund of Henan Polytechnic University (Grant No B2012-073), the Key Lab of Mine Informatization, Henan Polytechnic University (KY2015-08), and the Key Science and Technology Program of Henan Province (172102210274).

\section{References}

1. C. H. Bennett, G. Brassard, C. Crepeau, R. Jozsa, A. Peres, W. K. Wootters. "Teleporting an Unknown Quantum State Via Dual Classical and Einstein-Podolsky-Rosen Channels” [J]. Phys. Rev. Lett., 1993, 70.

2. D. Bouwmeester, J. W. Pan, K. Mattle, M. Eibl, H. Weinfurtor, A. Zeiling. "Experimental Quantum Teleportation" [J]. Nature, 1997, 390:575-579.

3. S. L. Braunstein, H. J. Kimble. "Teleportation of Continuous Quantum Variables" [J]. Phys. Rev. Lett., 1998, 80: 869-872.

4. W. J. Li, L. Song, W. C. Zhang, etc. "The Application Study of Equipment of Prevent Skip-Level Interview of Coal Mine Power Supply Based on Optic-Fibre Digital Communication" [J]. Embedded technology, 2011, 37(4): 26-32.

5. Y. C Luan, J. W. Liu. "Analysis and Discussion of Causes of Override Trip on Coal Mine” [J]. Industry and mine automation, 2009, (3): 82-84.

6. L. P. Shi, W. Y. Zhao, Z. M. Jiang, etc. "Scheme of Avoiding Override Trip in Mine Underground" [J]. Coal Mine Safety, 2012. 8: 115-117.

7. X. Y. Tian. "Fault Protection Override Trip Causes and Solutions of 10KV" [J]. Electrical Applications, 2008, 27(2): 31-33.

8. C. P. Yang, G. C. Guo. "Multiparticle Generalization of Teleportation" [J]. Chin. Phys. Lett., 2000, 17: $162-164$.

9. C. X. Yin, X. Q. Pan. "Studying on Override Trip of Coal Mine" [J]. Coal Science and Technology, 2011(6): 6669.

10. X. G. Zhang, H. M. Li, H. Ji, H. S. Zeng. "Controlled Teleportation of Multi-Qudit Quantum Information” [J]. Chin. Phys., 2007, 16.

11. S. B. Zheng. "Scheme for Approximate Conditional Teleportation of An Unknown Atomic State Without the BellState Measurement" [J]. Phys. Rev. A, 2004, 69.

12. Z. C. Zheng. "Research on Override Trip Causes of Tao er Coal Network"[J]. China Science and Technology Information. 2011, (15): 19-22.

13. N. R. Zhou, B. Y. Zeng, L. J. Wang, L. H. Gong. "Selective Automatic Repeat Quantum Synchronous Communication Protocol Based on Quantum Entanglement” [J]. Acta Phys. Sin, 2010, 59(4).

14. N. R. Zhou, G. H. Zeng, L. H. Gong, S. Q. Liu. "Quantum Communication Protocol for Data Link Layer Based on Entanglement” [J]. Acta Phys. Sin, 2007, 56(9): 55-58.

Xinliang Wang received his Ph.D. Degree in Signal and Information Processing from BeiJing University of Posts and Telecommunications in 2011. Now, he is an Associate Professor working in the School of Electrical Engineering and Automation at Henan Polytechnic University. His current research interests include smart power grids and coal mine high voltage power supply grids.

Zhigang Guo is a graduate student from the School of Physics and Electronic Information Engineering, Henan Polytechnic University. His research interests include smart power grids and coal mine high voltage power supply grids.

Qianhui Yang is a graduate student from the School of Electrical Engineering and Automation, Henan Polytechnic University. Her research interests include smart power grids and coal mine high voltage power supply grids.

Jianing Zou is a teacher working at the school of Physics and Electronic Information Engineering, Henan Polytechnic University. His current research interests include smart power grids and coal mine high voltage power supply grids. 